\title{
Voltage Regulation Considerations for the Design of Hybrid Distribution Transformers
}

\author{
Mohammed A Radi \\ Brunel Institute of Power Systems (BIPS) \\ Mohammed.Radi@Brunel.ac.uk
}

\author{
Mohamed Darwish \\ Brunel Institute of Power Systems (BIPS) \\ Mohamed.Darwish@Brunel.ac.uk
}

\author{
Mohammed Alqarni \\ Brunel University \\ eepgmar@brunel.ac.uk
}

\begin{abstract}
The future substation depends on finding a way to mitigate the effects of the drawbacks of the conventional legacy by employing the efficiency of the solid state switches [1]. This paper discusses the considerations of designing a distribution transformer that provides additional functions in regulating the voltage and controlling the reactive power that is injected in the distribution network, using a fractional rated converter attached partially with the windings of the transformer. This approach aims mainly to enhance the unit with more flexibility in controlling the voltage at the last mile of the network, in order to decrease the losses and meet the future expectations for low voltage networks modifications, and that by using a power electronic (PE) approach has less losses and more functionality (depending on the reliability of transformer and intelligence of PE). The design of a hybrid distribution transformer is detailed and its functionality in regulating the voltage is discussed as a combination between the features of one of the most reliable network devices, the transformer, and the effect of PE existence with less losses in both switching and conduction losses. Reduced ratings PE are used in this approach, whereby the solid state switches are controlled according to the immediate need for voltage control in low voltage $(\mathrm{LV})$ networks.
\end{abstract}

Index Terms -- Power electronics, Power systems, Substation transformers, Voltage regulation, Resonant controller, Low Voltage Networks, Power Quality.

\section{INTRODUCTION}

Future substation design depends on finding a way to employ the efficiency of the solid state switches of power electronics in the LV network that dispels the drawbacks of the traditional legacy system [1]. Electronic approaches will inform the acceptable choice for future hardware implementation of substation from a power electronics viewpoint, necessitating a tradeoff between flexibility, control function, efficiency and cost [1]. In distribution transformers, the ratio between the secondary and primary voltage is fixed and cannot be changed, where the use of the on-load tap changers (used in transformers) is limited, especially in the UK [2]. Poor voltage regulation is a direct reason for losses and shortening the life of several devices, whereas proper voltage regulation improves the quality of the delivered power. In low voltage conditions, the equipment works with a lower power factor and draws more current with constant power consumption, which means more losses in the feeders due to the relation $=I^{2} \mathrm{R}[2]$.

The designers of conventional substation transformers maintain the nominal voltage at the secondary side of the transformer to compensate the voltage drop accrued by the transformer and the impedance of the feeders. The tolerated voltage is usually $10 \%[2,3]$, as shown in Fig. 1 . If the voltage exceeds these limits, other devices and equipment might be damaged. Although the voltage is not supposed to exceed this limit according to regulations, increasing future demand and changing load types can cause increases. The nature of low voltage circuits differs from HV circuits, as low voltage circuits have a resistive nature more than inductive nature, which means that these circuits are capacity constrained by voltage regulation and not current flow [4].

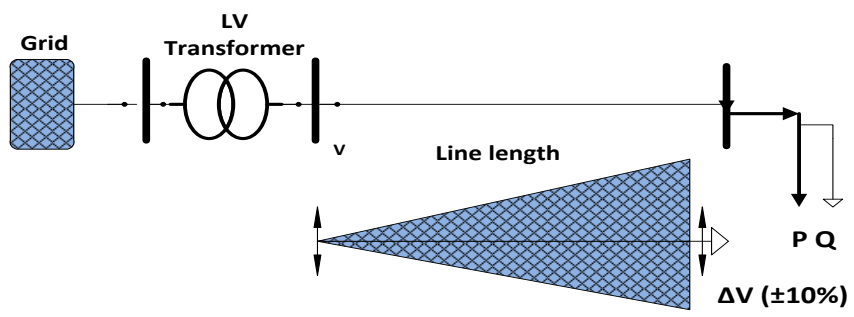

Fig. 1. Voltage drop behavior in LV networks

This paper proposes a voltage regulation approach for LV networks with a design based on the interaction between PE switches and the windings of the conventional transformer. The design is introduced as a hybrid distribution transformer, which is a voltage compensation approach that enhances the control at the low voltage side of the transformer (or the high voltage side in other configurations as in Ref [3]). The approach includes a distribution transformer with an attached fractionally rated $\mathrm{PE}$ converter, which contributes to supplying the transformer with additional controllability, and which could be used to control either voltage or reactive power compensation, or both of these functionalities in some control configurations as in [5].

\section{Voltage Regulation TeChNiQues}

Mechanical methods such as on load tap changers in distribution networks are not commonly used in voltage regulation in the $\mathrm{EU}$, especially the $\mathrm{UK}$, for the following reasons [6]:

- Frequent maintenance requirements for mechanical parts.

- High amount of losses during the changing process.

- The distribution network demands frequent voltage control actions during the day, which decreases the lifespan of the mechanical taps significantly.

- In distribution network, the ARC of the tap changer is close to the load. 
Voltage regulation is commonly understood and applied (e.g. in the UK) in terms of several fixed voltage boosts that are applied at different areas of the network; this provides for constant voltage decrease, which could be true these days but which will not be true for the different future scenarios of the network [7] and [8]. Typical voltage drops in the UK and compensations are illustrated in the following diagram.

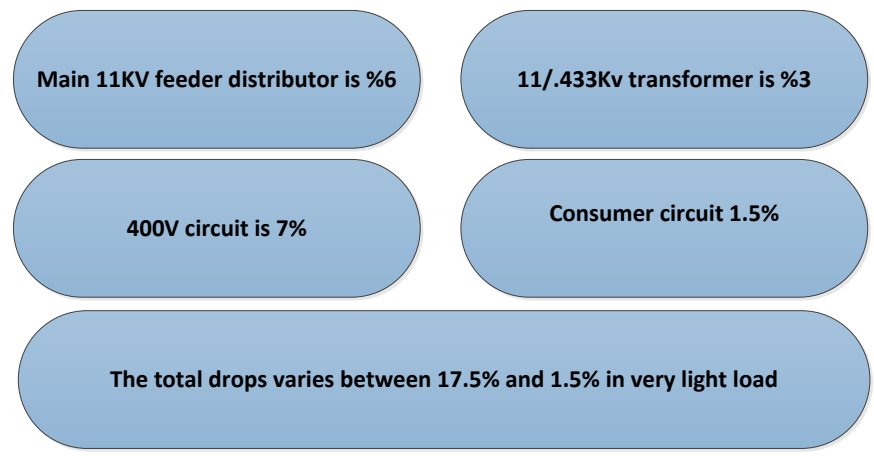

Fig. 2. Typical voltage drops in the distribution in UK

The older specification of " $240 \mathrm{~V}-10,+6 \%$ " means that the voltage has a limit of $216,254.4 \mathrm{~V}$. The revised specification (EN50160) says "230V -6+10\%", which equates to 216.2, $253 \mathrm{~V}$ [18]. To compensate for this drop, several voltage boosts are applied in different areas of the network, as shown below.

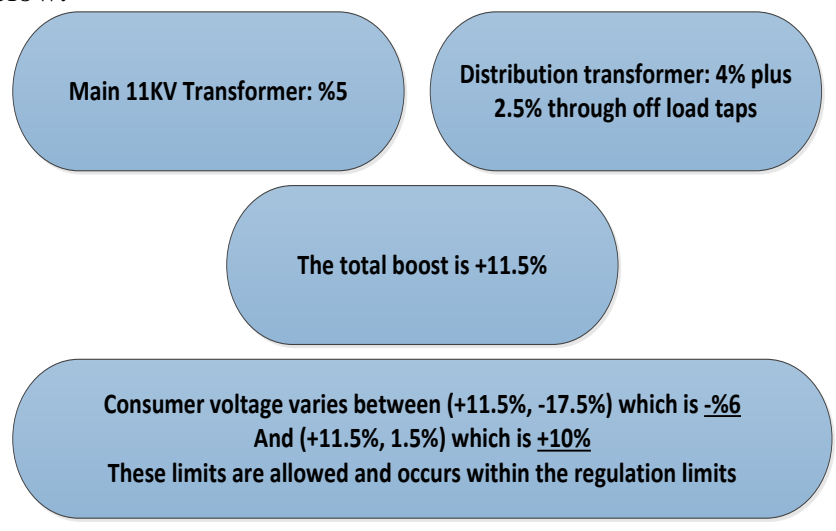

Fig. 3. The voltage level at the substation is installed high enough to compensate the voltage drop in the line under maximum load conditions

The distribution system has the transformer on one side and a large amount of customers on the other, which fundamentally unbalances the system; this is important and needs to be taken into account by voltage regulation studies. Power converter design should help to meet the requirements of the current and the future substations by operating interactively with other PE and conventional devices in the LV network to sustain continuous voltage regulation, voltage balance, and partial power factor correction with the aim of minimizing losses as much as possible [9].

\section{DESIGN AND APPROACH}

If only a $\pm 10 \%$ voltage limit for regulation is taken into consideration by the regulator, the switches of the $\mathrm{PE}$ converter can be designed at fractional ratings (around 10$20 \%$ ) of the total ratings of the LV transformer, which are the ratings that are needed to control the voltage regulation interval [3]. The following functionalities for the hybrid transformer could be achieved and considered according to its configurations [5]:

- Voltage regulation of up to $\pm 20 \%$.

- Reactive power control of up to \pm 20 .

- A combination of both topologies may be achieved as long as the total rating of the PE part is not exceeded.

- The attached converter can be protected by being bypassed in case of a failure within the system.

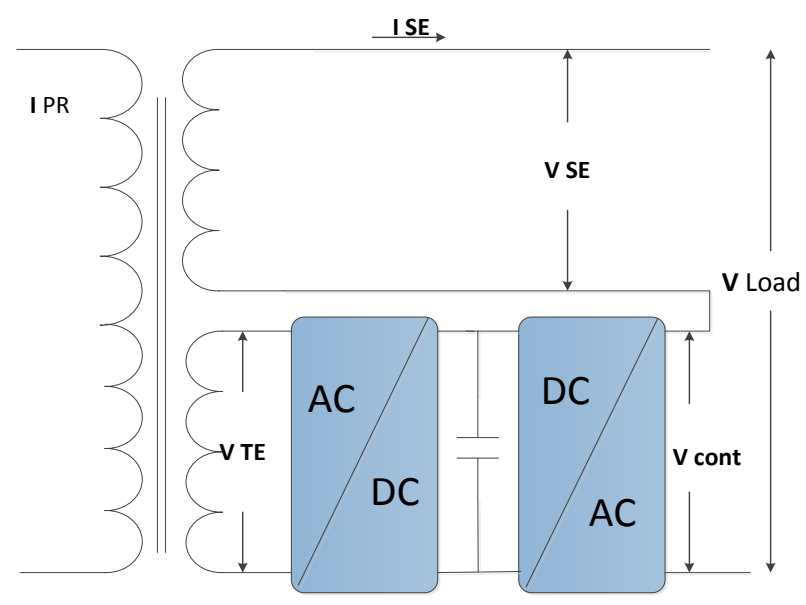

Fig. 4. AC regulation by using basic hybrid transformer [3]

The fractional rating of the attached converter provides the overall system with the following advantages:

- Lower cost and higher reliability compared to the full rated $\mathrm{PE}$

- Lower switching losses due to reducing the overall switches' ratings.

- The transformer is one of the most reliable devices in the network, thus its latent advantages are exploited.

- The system tastes partially the PE functionalities that could be bypassed in case of PE failure

Fractional power electronics are designed as a back-toback converter to control three unbalanced phases, each of which supplies three feeders to form nine lines that emerge from the transformer. Most feeders are made up of three phases and four wires, the latter of which is usually the neutral one. The three-phased feeder can usually range in length depending on load density, thus the introduced design takes into consideration an unbalanced system that needs voltage regulation for each phase separately [9], by attaching either three single-phase converters with the transformer as seen in Ref [10], or using a three-phase converter. 
The main function of the converter is to sustain a constant output voltage, to minimis the voltage fluctuations in the event of high or low demand.

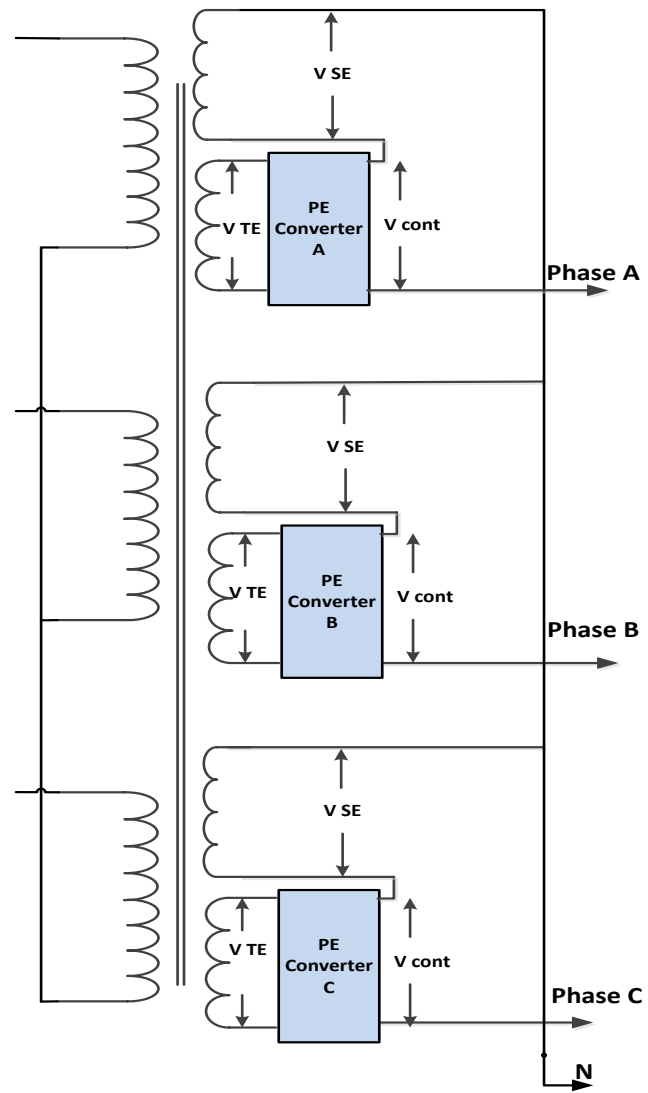

Fig. 5. The proposed hybrid transformer is introduced as a normal transformer that is attached partially with an AC/AC converter with a DC link [10].

Future scenarios require increasing attention to this function (e.g. increasing prevalence of electrical cars, whereby users plug and unplug their cars to charge frequently during the day), and it keeps the voltage and the current at the side of the transformer substantially in phase.

\section{TOPOLOGIES AND OPTIONS}

The different positions and various configurations for PE converters allow the transformer to perform various functions beside different techniques in regulating the voltage.

TABLE 1

DIFFERENT CONFIGURATIONS AND VARIOUS CAPABILITIES

\begin{tabular}{|l|l|}
\hline Conceptual schematics & \multicolumn{1}{|c|}{ Capabilities } \\
\hline PRR & $\begin{array}{l}\text { Option one has the ability to operate a } \\
\text { PF corrector for both displacement and } \\
\text { distortion PF (harmonic filter). It also } \\
\text { has an isolated DC source that could } \\
\text { operate through the bidirectional DC- } \\
\text { AC converter [5]. }\end{array}$ \\
\hline
\end{tabular}

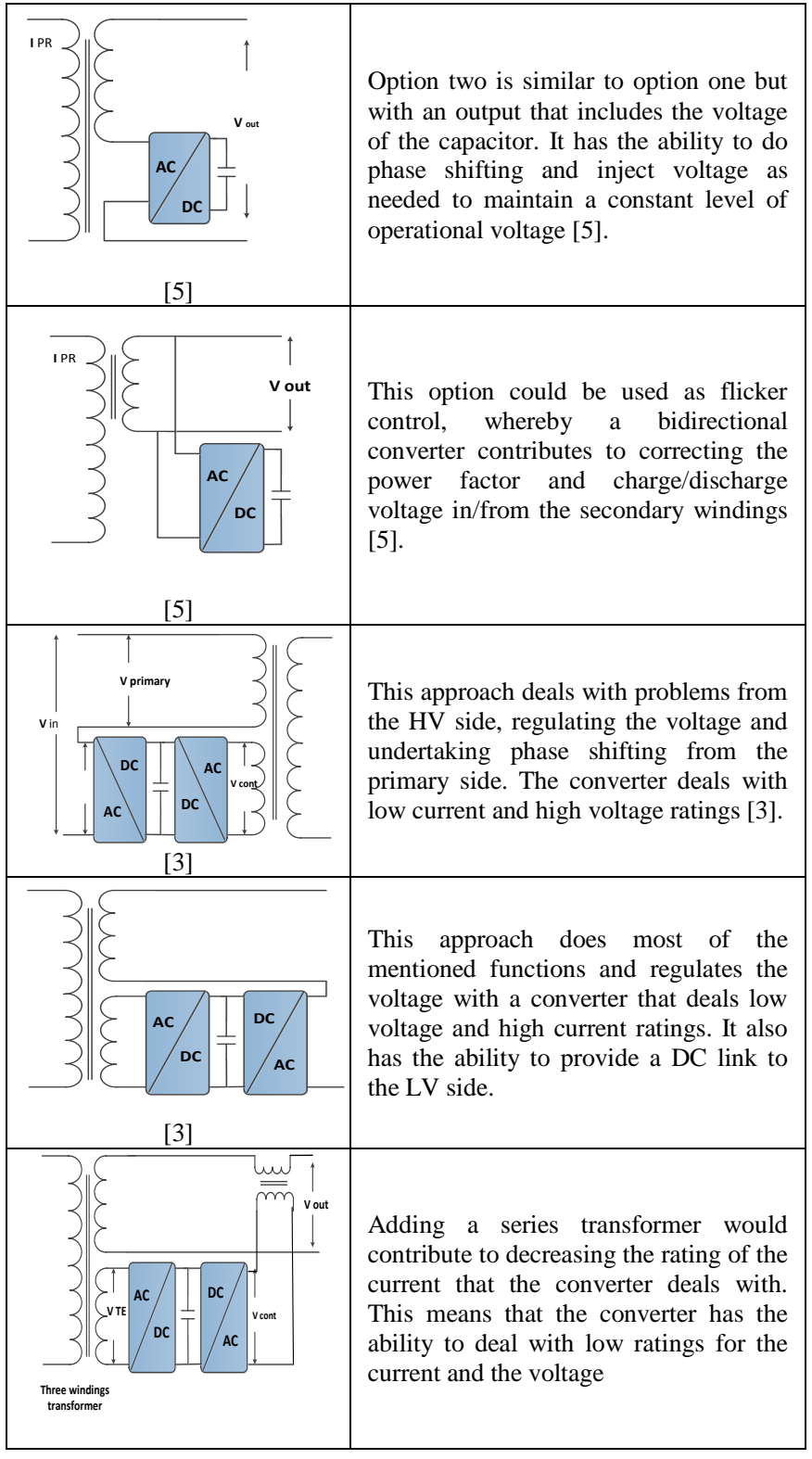

Every configuration has its own operational mode that provides the unit with different control abilities [5]. Possible conceptual schematics for the hybrid transformer are shown in Table 1, where every configuration has its own scheme in attaching the power electronics to the hybrid distribution transformer. The capability of each configuration is summarised in Table 1.

\section{CONTROL TOPOLOGY}

A resonant controller is taken into consideration to track a sinusoidal wave reference beside the need of controlling specific harmonic orders for a resistive load. Also, the $d q$ transformation technique is used to control the voltage at the DC link terminals [12], whereby the overall controller adds or decrease voltage $(10 \%-20 \%)$ to/from the total output voltage in order to control the whole output voltage of the transformer. 
The transformer ratios are [2]:

$$
\begin{gathered}
V_{\text {secondary }}=\frac{N 2}{N 1} \times V S \\
\mathrm{~V}_{\text {tertiary }}=\frac{\mathrm{N} 3}{\mathrm{~N} 1} \times \mathrm{Vs} \\
\mathrm{V}_{\mathrm{PE} \text { out }}=D \times V_{\text {tertiary }}
\end{gathered}
$$

Where $\mathrm{V}_{\mathrm{PE} \text { out }}$ is the output voltage of the converter, so $\mathrm{V}_{\text {out }}$ can be expressed as [2]:

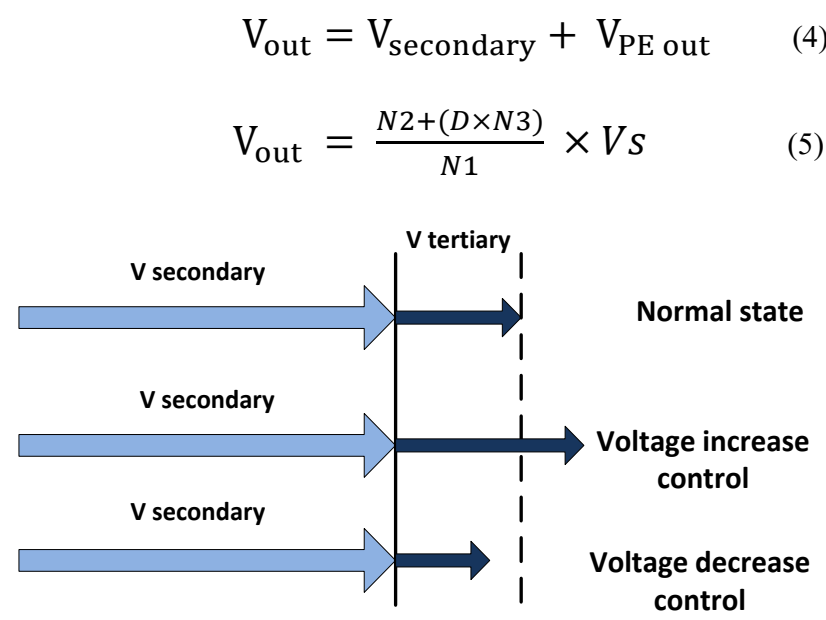

Fig. 6. Voltage control is applied in case of over voltage or under-voltage incidents at the output of the transformer; voltage control is chosen as a balance between the output of the secondary windings and the ac-ac converter [2].

The transformer supports a part of supplied voltage and the PE converter controls the other part of the voltage (voltage variations). If a voltage decrease occurs in the distribution line, the converter duty ratio correspondingly increases; conversely, when voltage increase occurs, the converter duty ratio decreases [11]. A vector diagram of voltage control is shown in Fig. 6.

\section{A. DC-link vector control}

A vector control is one of the most popular methods used for voltage source converter (VSC) [12].

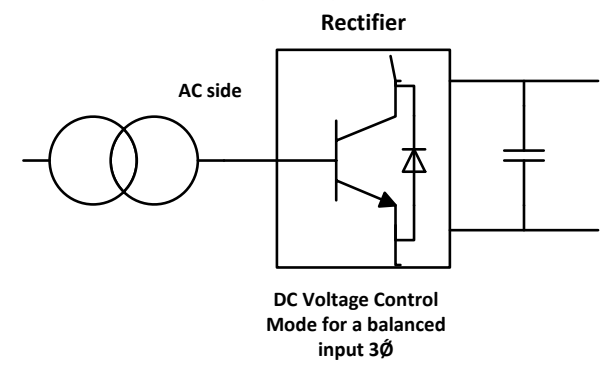

Fig. 7. Control structure of the VSC in the DC side [13]

Voltage and currents are described as vectors in the stationary $\alpha \beta$ and transformed after that to $d q$ coordinates to be controlled by two loops: inner loop for the current control and outer loop for the DC voltage control. The controlled coordinates are then transformed to feed the PWM generator in order to control the DC output of the converter. The inner loop transfer function and outer TF are stated in equations 6 and 7 respectively [13].

$$
G_{o . L}=K p \cdot\left(\frac{1+T i S}{T i S}\right)\left(\frac{1}{1+T e q S}\right) \cdot \frac{1}{r}\left(\frac{1}{1+\tau}\right)
$$

Where $\tau=\mathrm{L} / \mathrm{r}$

$$
G_{o . L}=K p \cdot\left(\frac{1+T i S}{T i S}\right)\left(\frac{1}{1+T e q S}\right)\left(\frac{3 V_{d}}{2 V_{d c}}\right) \cdot\left(\frac{1}{C S}\right)
$$

$T_{e q}=$ the time delay in the inner loop

An equivalent Laplace transformation for the circuits' material is done in order to determine the parameter of the PI controller. By this control the fluctuation in the DC link is minimised to enable fast and stable control for the AC side of the converter.

\section{B. Resonant control for the AC output voltage}

The normal $d q$ controller is appropriate for a balanced or slightly unbalanced system, whereby the transformation to dq frame can give an accepted performance, but in case of dealing with a severely unbalanced system the resonant controller (RC) is applied, as it is used in several approaches such as distribution generation and wind and solar energy.

In this paper the RC is chosen to control the voltage of the three phases separately by tracking a sinusoidal reference that takes its angle from phase locked loop (PLL) measurements from the network [14]. The resonant controller could be used also to eliminate specific harmonic orders beside controlling the output voltage as in Ref [15]. The proposed resonant control design is shown in Fig. 8; each phase is controlled separately due to the fact that each one is loaded differently.

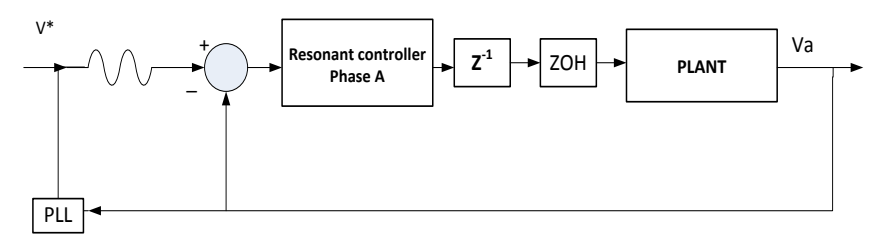

Fig. 8. Proposed resonant control diagram for each phase

The typical $\mathrm{TF}$ for the (RC) is in s-plan as shown in equation 9. The resonant frequency $\left(\omega_{0}\right)$ is the required frequency $(50 \mathrm{~Hz})[15]$.

$$
G_{C}(s)=K_{C} \frac{s^{2}+2 \zeta \omega_{n} s+\omega_{n}^{2}}{s^{2}+\omega_{o}^{2}}
$$

The s-plan transfer function is a transferee to z-plane, as in equation 10 [15].

$$
G_{C(z)}=K_{C z} \frac{z^{2}+a_{1}+a_{2}}{z^{2}+b_{1} z+b_{2}}
$$


The RC can be used also to eliminate a specific harmonics at specific frequencies by applying their frequencies in parallel implementations for the TF functions, as in equation 10 and Fig. 9. The control operates at the frequencies of the required harmonics that need to be eliminated [15], where $\omega_{o}=2 \pi f_{o}$.

$$
\begin{aligned}
& G_{C}(s)=K_{C} \frac{s^{2}+2 \zeta \omega_{n} s+\omega_{n}^{2}}{s^{2}+\omega_{o}^{2}}+K_{C a} \frac{s^{2}+2 \zeta \omega_{n a} s+\omega_{n a}^{2}}{s^{2}+\omega_{o}^{2} a}+ \\
& K_{c b} \frac{s^{2}+2 \zeta \omega_{n b} s+\omega_{n b}^{2}}{s^{2}+\omega_{o b}^{2}}+\ldots . K_{C m} \frac{s^{2}+2 \zeta \omega_{n m} s+\omega_{n m}^{2}}{s^{2}+\omega_{o m}^{2}}
\end{aligned}
$$

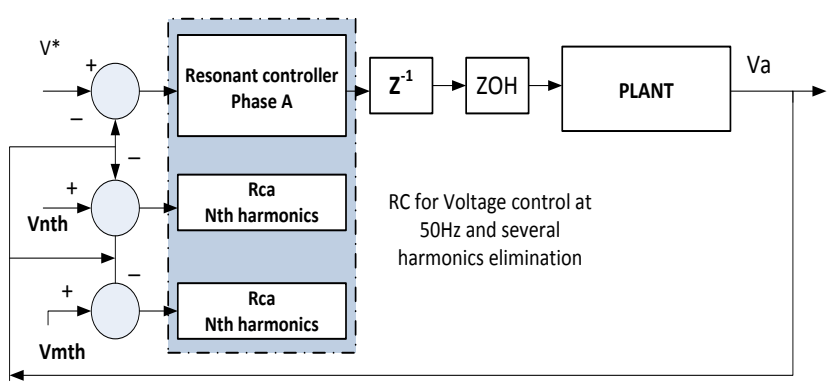

Fig. 9. RC for Voltage control at $50 \mathrm{~Hz}$ and several harmonics elimination

\section{FINDINGS AND RESULTS}

The DC link of the back-to-back converter is controlled by using a $d q$ transformation, as seen in Fig. 10.

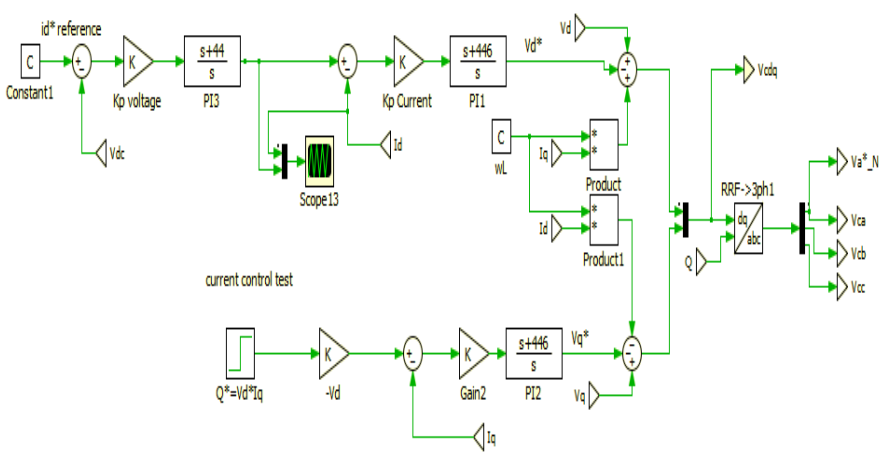

Fig. 10. $d q$ transformation technique

The control is used to maintain the DC voltage fixed at the terminal of the linked capacitor, in order to provide the $\mathrm{AC}$ part of the converter with a stable source of power [16]. The DC output responds to the reference of the controller and step up/down to the required value, as illustrated in Fig. 11.

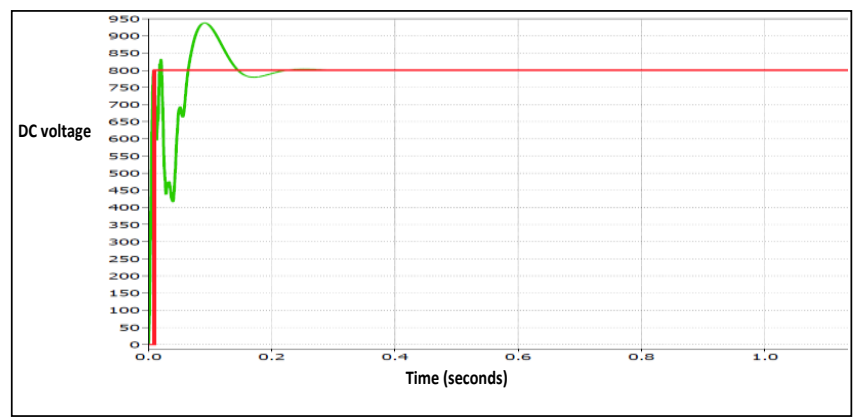

Fig. 11. Control response for the DC link
The AC side of the converter is controlled where the output of the converter is operating under several loading conditions, including voltage variations such as sags and short transient time [5]. LC filter is used to get a sinusoidal wave. Long time (sag) voltage regulation signal is illustrated below, before and after using the AC side of the transformer.

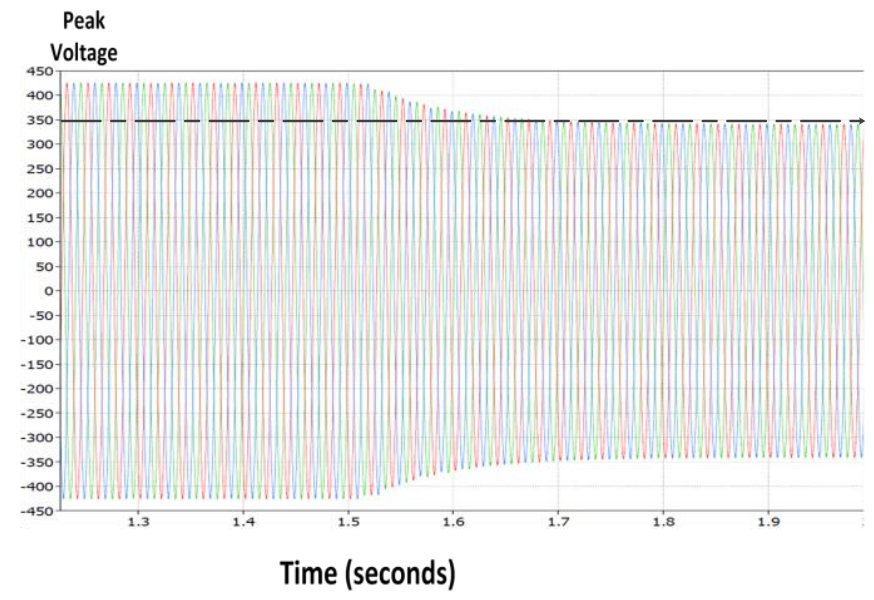

Fig. 12: Long time (sag) voltage regulation

This approach also provides the possibility of regulating the voltage over short transient time (second by second), as seen below in Fig. 13, where the reference of the voltage control is fixed and controlled in this case at the nominal voltage level, so the voltage regulation interval is decreased to be almost $\pm 0 \%$ instead of $\pm 10 \%$.

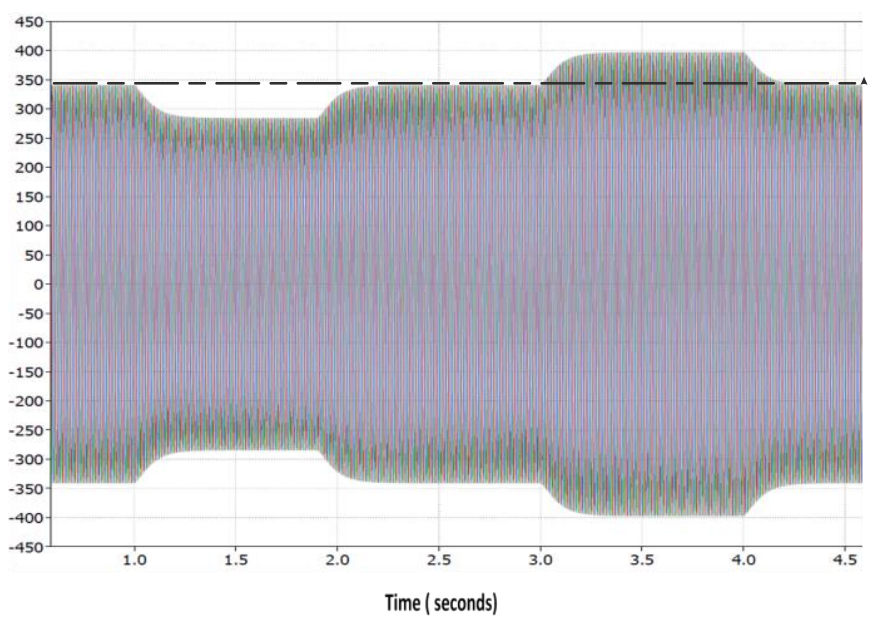

Fig. 13 Short transient time regulation

The voltage output is controlled during short and long time variations, which enhances the operation of sensitive voltage loads that require a firm and accurate voltage level, such as applications in medical equipment, where over-voltage can damage some electronic devices and reduce their operational life. On the other hand, decreasing the voltage can result in disabling the operation of these sensitive devices. The control of switches tries to provide a stable a DC link as a first level of control in order to isolate the variations of the input from the output, which ensures stable operation for the control at 
the AC side (final output), The DC link has the ability to be improved and used to provide a DC output source for a DC line or network in an enhanced form for the control, so it could operate synchronously between providing stability for the AC side and feeding a DC line (such as a DC network or charging a battery to be used in case of system failure) as a UPS approach as in Ref [17].

\section{CONCLUSION}

Owing to the expectations of increased load growth in future, the design of the LV substations entails specific requirements and improvements depending on the flexibility of PE in order to decrease the voltage variations and losses in LV network that increase by the time. The absence of tap control in 11/0.4 $\mathrm{kV}$ transformer addresses a voltage regulation problem: the existence of high voltage (HV) tap changers cannot help in case of load rich feeders in the last mile of the network. Voltage regulation could be feasible in the LV network by using PE functionality attached with the last point in the network [1] (before the loads point).

The hybrid distribution transformer is introduced as an approach that has the potential to upgrade the operation of the new LV substation to a new level that has the ability to meet the demand of the future distribution grid from an efficiency, controllability and volume perspective. Different conceptual schematics for the design of hybrid distribution transformer were introduced that have different abilities in serving several requirements in the $\mathrm{LV}$ network according to expected incidents of voltage variation scenarios and Var control [3]. Back-to-back converter was designed to represent the attached PE, which is controlled at two stages; the first stage uses $d q$ transformation for the purpose of fixing the voltage of the DC link, and the second stage aims to individually control each of the three phases at the AC output of the transformer using a resonant controller that has a sinusoidal voltage wave as a reference. The results of this have illustrated the converter's ability to control the voltage over short and long time voltage variations, which informs the choice of future substation hardware implementations by initializing a reasonable percentage of $\mathrm{PE}$ switches that operate with conventional reliable equipment, such as transformers.

\section{REFERENCES}

[1] EPSRC Top and Tail project proposal (the last mile electric network, Top and tail project, Nottingham University, 2011

[2] K. Yamaguchi, "Study on voltage regulation method in the power distribution system," Power Electronics, 2007. ..., pp. 488-492, 2007.

[3] J. Sastry and S. Bala, "Considerations for the design of power electronic modules for hybrid distribution transformers," ... Congress and Exposition (ECCE), 2013 IEEE, pp. 1422-1428, 2013.

[4] M. Kezunovic and Y. Guan, "The 21st century substation design: Vision of the future," Bulk Power System ..., pp. 1-8, Aug. 2010.

[5] S. Bala, D. Das, E. Aeloiza, A. Maitra, and S. Rajagopalan, "Hybrid distribution transformer: Concept development and field demonstration," 2012 IEEE Energy Conversion Congress and Exposition (ECCE), pp. 4061-4068, Sep. 2012.

[6] J. Faiz and B. Siahkolah, Electronic tap-changer for distribution transformers. 2011.
[7] H. a. Attia, "Optimal voltage profile control and losses minimization of radial distribution feeders," 2008 12th International Middle-East Power System Conference, pp. 453-458, Mar. 2008.

[8] H. E. Farag and E. F. El-Saadany, "Voltage regulation in distribution feeders with high DG penetration: From traditional to smart," 2011 IEEE Power and Energy Society General Meeting, pp. 1-8, Jul. 2011.

[9] L. Felber and H. Arango, "Voltage regulation in electric energy distribution substations," ... and Distribution ..., pp. 846-852, 2010.

[10] M. Haj-Maharsi and L. Tang, "Hybrid distribution transformer with ac \& dc power capabilities," US Patent App. 12/ ..., 2010.

[11] Das, R. P. Kandula, R. Harley, D. Divan, J. Schatz, and J. Munoz, "Design and testing of a medium voltage Controllable Network Transformer Prototype with an integrated hybrid active filter," 2011 IEEE Energy Conversion Congress and Exposition, pp. 4035-4042, Sep. 2011.

[12] T. L. Van and D.-C. Lee, "Developing Function Models of Back-toBack PWM Converters for Simplified Simulation," Journal of Power Electronics, vol. 11, no. 1, pp. 51-58, Jan. 2011.

[13] T. Kalitjuka, "Control of Voltage Source Converters for Power System Applications," Norwegian University of Science and Technology, 2011.

[14] Mihai Ciobotaru, Remus Teodorescu, Frede Blaabjerg, "A New Single-Phase PLL Structure Based on Second Order Generalized Integrator," Power Electronics and ..., 2007

[15] R. Cardenas and C. Juri, "The application of resonant controllers to four-leg matrix converters feeding unbalanced or nonlinear loads," ... , IEEE Transactions on, vol. 27, no. 3, pp. 1120-1129, 2012.

[16] S. Kalyani and G. Das, "Simulation of DQ control system for a unified power flow controller," ARPN Journal of Engineering and Applied ..., vol. 2, no. 6, pp. 10-19, 2007.

[17] Rasool and M. Darwish, "High frequency inverter circuit for UPS systems," Universities Power Engineering ..., pp. 1-4, Sep. 2012.

[18] Carbon Trust, "Voltage management: An introduction to technology and techniques." [Online]. Available: https://www.carbontrust.com/media/77191/ctg045.pdf. [Accessed: 04Apr-2014].

[19] Roshan and R. Burgos, "A DQ frame controller for a full-bridge single phase inverter used in small distributed power generation systems," Applied Power ..., pp. 641-647, 2007.

[20] Z. Fedyczak, J. Kaniewski, and M. Klyta, "Single-phase hybrid transformer using matrix-reactance chopper with Ćuk topology," Power Electronics and ..., 2007. 